\title{
Evaluación del dolor en la punción de una fístula arteriovenosa para hemodiálisis comparando pomada anestésica frente a frío local
}

\author{
Patricia Ahís Tomás*, Inma Peris Ambou*, Carmen Ma Pérez Baylach**, Joaquín Castelló Benavent*** \\ *Enfermeras, **Nefróloga. Centro de diálisis Nefrovall. La Vall D’Uixò. Castellón, ***Profesor de Matemáticas. \\ Universitat Jaume I. Castellón
}

\section{Resumen}

El dolor en pacientes de hemodiálisis no es valorado en su totalidad y no se tiene en cuenta las limitaciones que provoca en la calidad de vida de estos pacientes.

Se realizó un estudio observacional analítico, en el que se analizó el grado de dolor al que es sometido el paciente en hemodiálisis cuando se punciona su fístula arteriovenosa. Para ello comparamos el dolor que producía dicha punción si antes utilizábamos uno de estos tres métodos: POMADA ANÉSTESICA (Emla®), PLACEBO (crema hidratante) y HIELO. También hemos evaluado si el método utilizado interfiere en los parámetros de diálisis durante la sesión.

Estudiamos a 28 pacientes (23 hombres y 5 mujeres) portadores de fístula. Las variables medidas fueron: dolor (escala EVA), flujo sanguíneo, presión arterial, presión venosa y tiempo de coagulación. Estas variables fueron recogidas durante las tres semanas de duración del estudio (36 sesiones de hemodiálisis) en gráficas.

Se analizaron las 36 sesiones de hemodiálisis mediante el uso de una gráfica. Por un lado observamos que el método elegido $(E m l a ß)$ es estadísticamente significativo ( $p$-valor, $p=0.004$ ), por otro lado no existen diferencias significativas entre el método usado y el resto de parámetros recogidos durante la sesión de hemodiálisis $(p=0.194, p=0.278, p=0.902, p=0.445)$. Con respecto al método utilizado los pacientes eligieron como mejor método la Emla ${ }^{\circledR}$ con un $67.86 \%$.

Según los datos obtenidos podemos concluir que no existen diferencias estadísticas en las diferentes variables estudiadas. El uso de Emla ${ }^{\circledR}$ obtiene mejor resultado en cuanto al dolor, pero no varía ningún otro parámetro al igual que los otros dos métodos.

$$
\begin{gathered}
\text { Correspondencia: } \\
\text { Patricia Ahís Tomás } \\
\text { Centro de Hemodiálisis Nefrovall } \\
\text { Pol. Ind. Uxolanz,6. (12600) } \\
\text { La Vall D’Uixò. Castellón } \\
\text { E-mail: patriahis@hotmail.com }
\end{gathered}
$$

\author{
PALABRAS CLAVE: \\ - DOLOR \\ - PUNCIÓN FÍSTULA ARTERIOVENOSA \\ - EMLA \\ - DIÁLISIS
}

Pain assessment in an arteriovenous fistule puncture for hemodialysis using anaesthetic cream vs ice

\section{Abstract}

Pain in hemodialysis patients is not valued in its entirety and does not take into account the limitations resulting in the quality of life of these patients.

We performed an observational study, which analyzed the degree of pain, that suffers a patient undergoing hemodialysis, when is punctured his arteriovenous fistule. To do this we compare the pain that occurred before the puncture if we used one of three methods: anaesthetic cream (Emla $®$ ), PLACEBO (moisturizer) and ICE. We also assessed whether a method interferes dialysis parameters during the session.

We studied 28 patients ( 23 men and 5 women) carrying fistula. The variables measured were pain (VAS), blood flow, blood pressure, venous pressure and clotting time. These variables were collected during the three-week duration of the study (36 sessions of hemodialysis) graphs.

36 were analyzed hemodialysis sessions using a graph on one hand we observed that the used method is statistically significant ( $p$ - value, $p=0.004$ ), on the other hand, no significant differences between the used method and the remaining parameters contained in the hemodialysis session $(p=0.194, p=0.278, p=0.902$, $p=0.445$ ). With regard to the used method patients chose as best method Emla $®$ with $67.86 \%$.

According to the data we can conclude that there are no statistical differences in the studied variables. Emla ${ }^{\circledR}$ using gets better result in terms of pain, but does not change any other parameters like the other two methods. 


\section{KEY WORDS:}

- PAIN

- FISTULE NEEDLE ARTERIOVENOUS

- EMLA

- DIALYSIS

\section{Introducción}

El dolor es una sensación subjetiva de malestar o sufrimiento que se origina como resultado de estimulaciones nocivas que indican daño tisular o enfermedad de cualquier tipo ${ }^{1}$. El paciente sometido a hemodiálisis, lleva asociado a su enfermedad una gran comorbilidad que a menudo le causan dolor. Generalmente este dolor no es valorado en su totalidad y no se tienen en cuenta las limitaciones que provoca en la calidad de vida de estos pacientes.

El acceso vascular es uno de los elementos claves en el manejo de los pacientes con insuficiencia renal crónica terminal en programa de hemodiálisis (HD) periódica y en la aceptación de la diálisis por parte del paciente. El acceso vascular de elección en estos pacientes es la fístula arteriovenosa (FAVI) ${ }^{2}$.

La punción periódica de la FAVI ocasiona un grado de dolor considerable, debido al gran calibre y longitud del bisel de las agujas para la fístula, ocasionando molestias y disconfort al paciente ${ }^{3,4,5}$.

Los pacientes que reciben el tratamiento de diálisis son sometidos a dos punciones diarias con agujas de calibre elevado (15G), tres veces por semana.

Para nosotros es importante poder valorar el grado de dolor de nuestros pacientes y con ello poder disminuirlo.

Nos planteamos como hipótesis de estudio que las punciones en la FAVI, tras el uso de método tópico anestésico Emla® y hielo producen menor dolor que aquellas en las que no se usan estos métodos.

Por ello nos fijamos como objetivo principal comparar el grado de dolor al que es sometido el paciente en hemodiálisis cuando se punciona su fistula arteriovenosa (FAVI). Para ello compararemos el dolor que produce dicha punción si se usa antes POMADA ANÉSTESICA (Emla®), PLACEBO (crema de manOS) O HIELO.

\section{Material y métodos}

Diseñamos un estudio observacional analítico.

Se incluyeron a los pacientes portadores de FAVI (39), que tras aplicar los criterios de exclusión (pacientes con hipersensibilidad conocida al anestésico local, pacientes que estén sometidos a tratamiento con medicamentos antiarrítmicos clase III, pacientes ingre-

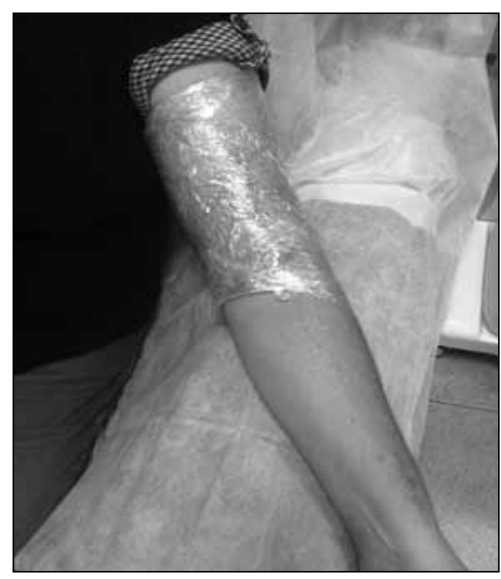
sados durante el estudio, pacientes que utilicen otros métodos para el alivio del dolor, pacientes que se nieguen a participar y pacientes que presenten demencia). Previamente se informó a los pacientes en qué consistía nuestro estudio y todos los pacientes incluidos en el estudio firmaron el consentimiento informado. (Para identificar a los pacientes se uso un número de registro que aparece en nuestra base de datos, con ello se consigue total confidencialidad).

La duración del estudio fue de tres semanas. Durante la primera semana se utilizó la pomada anestésica $\left(\right.$ Emla $\left.{ }^{\circledR}\right)$, la segunda semana crema hidratante (Placebo) para finalizar la tercera semana se aplicó hielo. Los pacientes no tenían conocimiento de qué tipo de pomada se les estaba aplicando. Para cada método usado se realizaron tres protocolos de aplicación.

Se realizó un taller para que los pacientes tuvieran conocimiento de cómo administrarse la Emla ${ }^{\circledR}$ en sus domicilios mediante una demostración en cada grupo.

Las punciones efectuadas a un mismo paciente no se realizaron por el mismo profesional, debido al ratio de pacientes que tiene cada enfermero del centro. En lo referente al calibre de las agujas se uso el mismo para todos (15G).

No obstante se intentó durante el es-

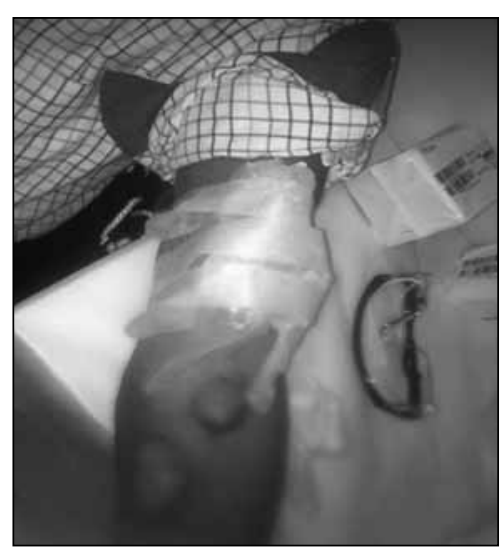


tudio no puncionar zonas nuevas o poco puncionadas, es decir, se utilizaron las zonas habituales.

Como instrumento de medida hemos usando la escala del dolor EVA, es una escala numérica del 0 al 10, donde " 0 " es ausencia de dolor y "10" dolor insoportable.

Para otras valoraciones se ha diseñado una hoja de recogida de datos (Anexo $\mathbf{I}$ ) donde se registraron los siguientes parámetros: problemas de punción (sí o no), numero de agujas $(2,3$ o 4), flujo sanguíneo $(<300$ >300), facilidad en la punción (más fácil, igual o más difícil), reacciones adversas (donde se indica si ha aparecido alguna de la lista), tiempo de coagulación $(5,10$, $15,>15$ minutos) y si apareció alguna variación en el tacto (sí o no).

Para el análisis estadístico se introdujeron los datos apuntados en la hoja de registro en una tabla de Excel y posteriormente se exportaron al programa de análisis estadístico R-Commander. Se aceptó significación estadística para $p$-valor $<0.05$.

\section{Resultados}

La muestra la formaron un total de 28 pacientes portadores de FAVI, donde el $82 \%$ son hombres. Las edades fueron comprendidas entre 38+-90. El $80 \%$ de los pacientes reciben tratamiento desde hace más de 5 años. Se analizaron las 36 sesiones de hemodiálisis mediante el uso de una gráfica donde se registraron los resultados de todas las variables. Por un lado observamos que el método usado es estadísticamente significativo ( $p=0.004$ ), por otro lado no existen diferencias significativas entre el método usado y el resto de parámetros recogidos durante la sesión de hemodiálisis (PV $p=0.191$, PA $p=0.278$, FLUJ0 $p=0.902$, TCO $p=$ $0.445)$, en las tablas se pueden observar los diferentes valores obtenidos en los tres métodos usados durante el estudio (tabla I, II y III).

Con respecto al método utilizado los pacientes eligieron como mejor la Emla ${ }^{\circledR}$ con un $67.86 \%$. (Gráfica I).
Tabla I. Valores obtenidos con el uso de EMLA®.

\begin{tabular}{|l|c|}
\hline \multicolumn{1}{|c|}{ Variable (Emla®) } & Media \\
\hline Escala EVA & 2 \\
\hline Problemas en la punción & NO \\
\hline$N^{\circ}$ de agujas & 2 \\
\hline Flujo sanguíneo & 340 \\
\hline Peor tacto & NO \\
\hline Facilidad en la punción & IGUAL \\
\hline Presión arterial & 164 \\
\hline Presión venosa & 151 \\
\hline Tiempo de coagulación & 13 \\
\hline Reacciones adversas & NO \\
\hline
\end{tabular}

Tabla II. Valores obtenidos con el uso del placebo.

\begin{tabular}{|l|c|}
\hline Variable (Placebo) & Media \\
\hline Escala EVA & 4.40 \\
\hline Problemas en la punción & NO \\
\hline $\mathrm{N}^{\circ}$ de agujas & 2 \\
\hline Flujo sanguíneo & 365 \\
\hline Peor tacto & NO \\
\hline Facilidad en la punción & IGUAL \\
\hline Presión arterial & 167 \\
\hline Presión venosa & 150 \\
\hline Tiempo de coagulación & 14 \\
\hline Reacciones adversas & NO \\
\hline
\end{tabular}

Tabla III. Valores obtenidos con el uso del hielo.

\begin{tabular}{|l|c|}
\hline Variable (Hielo) & Media \\
\hline Escala EVA & 4.70 \\
\hline Problemas en la punción & $\mathrm{NO}$ \\
\hline $\mathrm{N}^{0}$ de agujas & 2 \\
\hline Flujo sanguíneo & 339 \\
\hline Peor tacto & $\mathrm{NO}$ \\
\hline Facilidad en la punción & $\mathrm{IGUAL}$ \\
\hline Presión arterial & 166 \\
\hline Presión venosa & 144 \\
\hline Tiempo de coagulación & 14 \\
\hline Reacciones adversas & ROJEZ \\
\hline
\end{tabular}

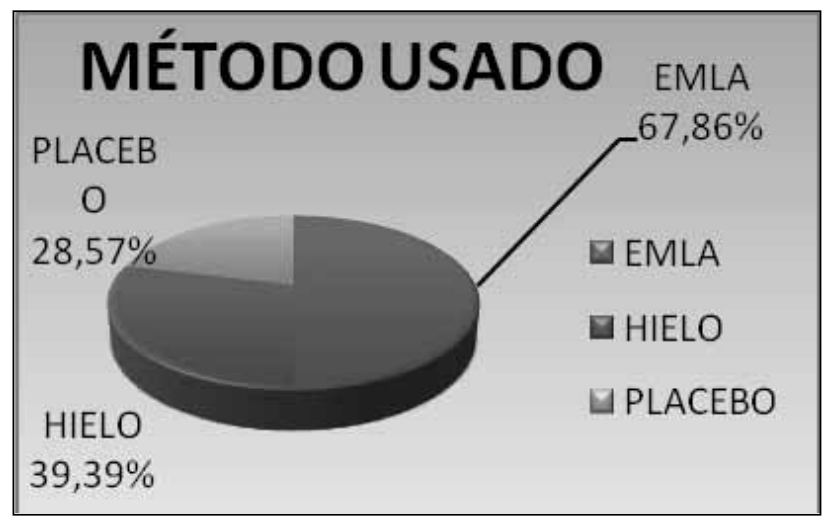

Gráfica I. Método elegido por los pacientes. 


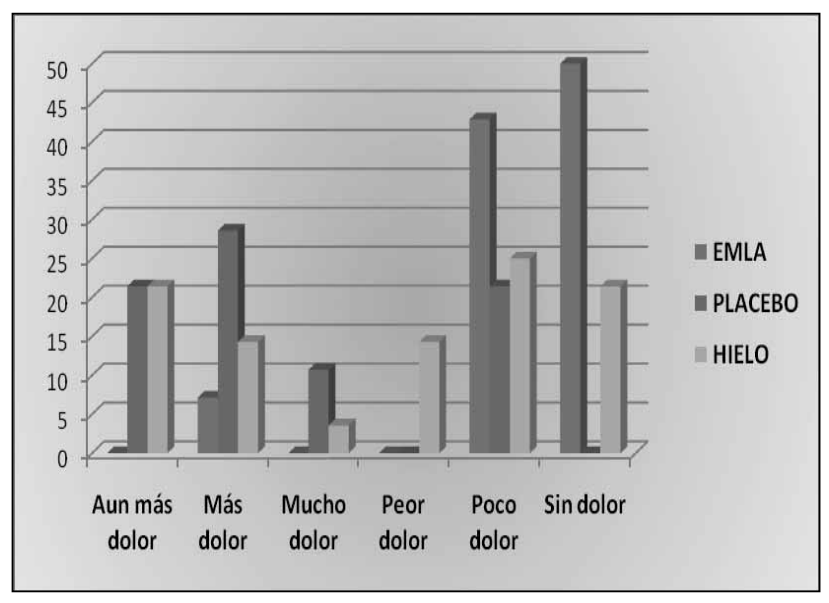

Gráfica II. Evaluación del dolor.

Según los datos obtenidos podemos concluir que no existen diferencias estadísticas entre las diferentes variables estudiadas. El uso de Emla® obtiene mejor resultado en cuanto al dolor (Gráfica II), pero no varía ningún otro parámetro al igual que los otros dos métodos.

\section{Discusión}

El dolor provocado por la punción de la FAVI es un factor importante tanto para el paciente como para el profesional de enfermería, ya que los pacientes son sometidos a tres punciones semanales provocándoles en muchas ocasiones sensación de malestar, preocupación y miedo.

En la actualidad no hay muchos estudios que tengan por objetivo valorar este tema y es, sin embargo, uno de los temas que más preocupan a nuestros pacientes a la hora de aceptar la fístula arteriovenosa como acceso vascular. En la bibliografía sí que hemos encontrado estudios con Emla ${ }^{\circ}$ e incluso el uso de agujas congeladas, buscando todos ellos amortiguar el dolor que producen las punciones $^{1,6}$. También valoramos el uso de placebo para valorar sí realmente el empleo de crema anestésica es efectivo. Con estos datos decidimos comparar estos 3 métodos de uso tópico con el propósito de mejorar la calidad de la diálisis. Nuestros pacientes eligieron de forma significativa la pomada anestésica respecto a los otros 2 métodos, aunque el uso de esta es efectivo pero no elimina por completo la sensación de dolor. El uso del hielo que creíamos mitigaría el dolor no resultó efectivo. No sabemos si el hecho de realizar el estudio en invierno facilito el rechazo a este método dado que este provocaba disconfort. Tras el estudio muchos pacientes han comenzado con el uso de crema anestésica antes de venir a sus sesiones y nos consta que su calidad de vida en este aspecto ha mejorado.
En la bibliografía consultada, se relaciona el uso de la pomada con procedimientos únicos y en situaciones agudas como pruebas diagnosticas y canalizaciones de catéteres que se diferencian de un proceso crónico como es el caso de la HD ${ }^{7,8}$. En los procesos crónicos, las diferencias en la percepción de dolor, favorecen una ampliación del estudio.

\section{Agradecimientos}

A todos los compañeros del centro (Médicos, Enfermeras y Auxiliares) por haber colaborado y ayudado durante el estudio.

A todos los enfermos que han participado, sin su colaboración e interés no hubiera sido posible este estudio.

Recibido: 14 Septiembre 2013

Revisado: 27 Noviembre 2013

Modificado: 24 Enero 2014

Aceptado: 10 Febrero 2014

\section{Bibliografía}

1. Cañada Alvarez R, Puig Plá J, Ferrero Hidalgo S. Grado de dolor al pinchar la fístula arteriovenosa con agujas congeladas comparando con agujas a temperatura ambiente. Rev. Soc. Esp. Enferm. Nefrol. $2004 ; 7(2)$ : 74-76.

2. Crespo $R$, Casas $R$, Muñoz J, Rivero $F$, Contreras $M^{a} D$, Muñoz $L$, López $E$. Influencia del calibre de la aguja sobre el grado de dolor originado en la punción de la fístula arteriovenosa. Rev. Soc. Esp. Enferm. Nefrol. 2002; 119-124.

3. Maciá A, Pina JA. Recomendación de pomada anestésica emla en la punción a pacientes sometidos a hemodiálisis periódica. Revista científica de Enfermería RECIEN. 2010;1989-6409.

4. García J, Sousa F, Rodríguez $T$, Andrés J, Alcalde S, Cardeñoso E, de la Cruz V, Hera R, Martín B, Ovejero C, Prieto V, Villacorta T, Carrera. Aplicación tópica de la lidocaína-prilocaína (emla) en pacientes en hemodiálisis ¿Disminuye el dolor en la punción de la fístula? Libro de comunicaciones del XXV Congreso de la SEDEN; Oviedo, 2000, 7-12. 
5. Granados Navarrete I, Abril Sabater D, Alcaraz Busqueta $F$, Mañé Buixo N, Padilla Ruiz J, Real Gatius J, Solano Pallarés M, Yuste Giménez E Una actuación de enfermería: intentar aliviar el dolor en las punciones de hemodiálisis. Rev. Soc. Esp. Enferm. Nefrol. 2005; 8 (3): 231-236.

6. Villanueva A, Cancela R. EMLA Revisión de la literatura. Rev Cent Dermatol Pascua, Ene-Abr 1999;vol. 8 (Núm. 1):12-15.
7. Berná-Serra JD, Redondo MV, Durán I, BernáMestre JD. Galactography without discomfort using lidocaine/prilocaine anesthesic cream. Acta Radiol, 2008; 49 (1):22-4.

8. Pirat $A$, Karaaslan $P$, Cadan $S$, Zeyneloglu $P$, Varan B, Tokel K, Torgay A, Arslan G. Topical EMLA cream versus prilocaine infiltration for pediatric cardiac catheterization. J ardiothorac Vasc Anesth. 2005 0ct; 19 (5):642-5.

ANEXO IV. Hoja de Registro.

NÚMERO DE REGISTRO:

\begin{tabular}{|c|c|c|c|c|c|c|c|c|c|c|c|}
\hline \multirow[t]{2}{*}{ FECHA: } & \multicolumn{2}{|c|}{$1^{\circ}$ SESIÓN } & \multicolumn{3}{|c|}{$2^{\circ} \mathrm{SESION}$} & \multicolumn{3}{|c|}{$3^{\circ}$ SESION } & & & \\
\hline & $\begin{array}{c}\text { Escala } \\
\text { EVA }\end{array}$ & $\begin{array}{c}\text { Problemas } \\
\text { punción }\end{array}$ & en la & $\begin{array}{c}\mathbf{N}^{\circ} \\
\text { agujas }\end{array}$ & Flujo & sanguíneo & Peor & Tacto & Facilidad & en la & punción \\
\hline $1^{\circ}$ SESIÓN EMLA ${ }^{1}$ & & SI & NO & & $<300$ & $>300$ & SI & NO & Más fácil & Igual & Más difícil \\
\hline $2^{\circ}$ SESIÓN EMLA & & SI & NO & & $<300$ & $>300$ & SI & NO & Más fácil & Igual & Más difícil \\
\hline $3^{\circ}$ SESIÓN EMLA & & SI & NO & & $<300$ & $>300$ & SI & NO & Más fácil & Igual & Más difícil \\
\hline
\end{tabular}

\begin{tabular}{|l|c|c|c|c|c|c|c|c|}
\hline & Presión & arterial & Presión & venosa & Tiempo & De & coagulación \\
\hline $\mathbf{1}^{\mathbf{0}}$ SESIÓN EMLA & $100-150$ & $150-200$ & $<200$ & $\geq 200$ & 5 & 10 & 15 & $>15$ \\
\hline $\mathbf{2}^{\circ}$ SESIÓN EMLA & $100-150$ & $150-200$ & $<200$ & $\geq 200$ & 5 & 10 & 15 & $>15$ \\
\hline $3^{\circ}$ SESIÓN EMLA & $100-150$ & $150-200$ & $<200$ & $\geq 200$ & 5 & 10 & 15 & $>15$ \\
\hline
\end{tabular}

\begin{tabular}{l|l|l|l|l|l|l|}
$\begin{array}{l}\text { REACCIONES } \\
\text { ADVERSAS }\end{array}$ & PALIDEZ & ENROJECIMIENTO & HINCHAZÓN & $\begin{array}{c}\text { SENSACIÓN INICIAL } \\
\text { DE QUEMAZÓN }\end{array}$ & PICOR & OTRAS \\
\hline $1^{\circ}$ SESIÓN & & & & & \\
\hline $2^{\circ}$ SESIÓN & & & & & \\
\hline $3^{\circ}$ SESIÓN & & & & \\
\hline
\end{tabular}

1 Se cambió el método cada semana (Emla ${ }^{\circledR}$, placebo y hielo).

2 Señalar con una cruz si aparece esa reacción adversa, sino dejar la casilla en blanco. 